PROCEEDINGS OF THE

AMERICAN MATHEMATICAL SOCIETY

Volume 135, Number 1, January 2007, Pages 59-68

S 0002-9939(06)08730-2

Article electronically published on August 16, 2006

\title{
ON A SEMILINEAR PARABOLIC EQUATION
}

\author{
LOTFI RIAHI
}

(Communicated by David S. Tartakoff)

\begin{abstract}
We introduce a general class of potentials $V=V(x, t)$ so that the semilinear parabolic equation $a \Delta u-\frac{\partial}{\partial t} u+V u^{p}=0$ in $\left.\mathbb{R}^{n} \times\right] 0, \infty[, n \geq 3, p>$ $1, a>0$, has global positive continuous solutions. These results extend the recent ones proved by Zhang to a more general class of potentials.
\end{abstract}

\section{INTRODUCTION}

In this paper we will be interested in the following semilinear parabolic problem:

$$
\left\{\begin{array}{l}
\left.a \Delta u-\frac{\partial}{\partial t} u+V(x, t) u^{p}=0 \quad \text { in } \mathbb{R}^{n} \times\right] 0, \infty[, \\
\left.u>0 \text { in } \mathbb{R}^{n} \times\right] 0, \infty[ \\
u(x, 0)=u_{0}(x), \quad x \in \mathbb{R}^{n}
\end{array}\right.
$$

where $n \geq 3, p>1, \Delta$ is the Laplacian operator and $V \in L_{\text {loc }}^{1}\left(\mathbb{R}^{n} \times \mathbb{R}\right)$. We first recall that Zhang [5] studied the global existence for this problem when the potential $V$ is in the parabolic Kato class at infinity $P^{\infty}$, and in the time-independent case when $V=V(x)$ is in the subclass of Green tight functions, the asymptotic behavior of solutions for this problem is studied by Zhang and Zhao [6]. The elliptic counterpart problem $\Delta u(x)+V(x) u^{p}(x)=0, x \in \mathbb{R}^{n}, n \geq 3$, was studied by some authors during the last two decades (see Kenig and Ni [2], Lin [3], Ni 4], Zhao [7] and the references therein).

Our aim in this paper is to introduce a new class of potentials $V$ denoted by $P_{\text {loc }}^{\infty}$ so that the problem $\left(\mathcal{P}_{V}\right)$ has a global continuous solution comparable to that of $\left(\mathcal{P}_{0}\right)$. We also prove that when $V \geq 0$, the condition $V \in P_{\mathrm{loc}}^{\infty}$ is optimal for this result to hold. In particular, we extend the results proved by Zhang [5] to a new functional class more general than the parabolic Kato class $P^{\infty}$. We also give interesting examples of potentials in $P_{\mathrm{loc}}^{\infty} \backslash P^{\infty}$.

Let us introduce the class $P_{\mathrm{loc}}^{\infty}$.

Received by the editors June 30, 2004.

2000 Mathematics Subject Classification. Primary 35J60, 35K55.

Key words and phrases. Parabolic equation, positive solution, fixed point theorem.

(C)2006 American Mathematical Society Reverts to public domain 28 years from publication 
Definition 1.1. A Borel measurable function $V=V(x, t)$ on $\mathbb{R}^{n} \times \mathbb{R}$ is said to be in the class $P_{\mathrm{loc}}^{\infty}$ if it satisfies for all $c>0$,

$$
\begin{aligned}
N_{c}(V) \equiv & \sup _{(x, t) \in \mathbb{R}^{n} \times \mathbb{R}} \int_{-\infty}^{t} \int_{\mathbb{R}^{n}} \Gamma_{c}(x, t ; y, s)|V(y, s)| d y d s \\
& +\sup _{(y, s) \in \mathbb{R}^{n} \times \mathbb{R}} \int_{s}^{+\infty} \int_{\mathbb{R}^{n}} \Gamma_{c}(x, t ; y, s)|V(x, t)| d x d t<\infty,
\end{aligned}
$$

and for any compact subset $A \subset \mathbb{R}^{n} \times \mathbb{R}$,

$$
\begin{aligned}
\lim _{r \rightarrow 0} & \left\{\sup _{(x, t) \in A} \int_{t-r}^{t} \int_{|x-y|<\sqrt{r}} \Gamma_{c}(x, t ; y, s)|V(y, s)| d y d s\right. \\
& \left.+\sup _{(y, s) \in A} \int_{s}^{s+r} \int_{|x-y|<\sqrt{r}} \Gamma_{c}(x, t ; y, s)|V(x, t)| d x d t\right\}=0,
\end{aligned}
$$

where $\Gamma_{c}(x, t ; y, s)=\frac{1}{(t-s)^{n / 2}} \exp \left(-c \frac{|x-y|^{2}}{t-s}\right)$, for $t>s$.

Let $P^{\infty}$ be the class introduced by Zhang in [5]. Obviously $P^{\infty} \subset P_{\mathrm{loc}}^{\infty} \subset$ $L_{\text {loc }}^{1}\left(\mathbb{R}^{n} \times \mathbb{R}\right)$ and we have the following.

Remark 1.1. Let $V$ be a Borel measurable function on $\mathbb{R}^{n} \times \mathbb{R}$ satisfying $N_{c}(V)<\infty$, for all $c>0$. Then $V \in P_{\text {loc }}^{\infty}$ if and only if $V \varphi \in P^{\infty}$ for all $\varphi \in C_{0}^{\infty}\left(\mathbb{R}^{n} \times \mathbb{R}\right)$.

In the time-independent case we have the following.

Remark 1.2. Let $\mathcal{M}_{b}$ be the set of functions $V=V(x)$ on $\mathbb{R}^{n}$ which define bounded Newtonian potentials, i.e. $\sup _{x \in \mathbb{R}^{n}} \int_{\mathbb{R}^{n}} \frac{|V(y)|}{|x-y|^{n-2}} d y<\infty$. Then $P^{\infty}=K_{n} \cap \mathcal{M}_{b}$ and $P_{\text {loc }}^{\infty}=K_{n}^{\text {loc }} \cap \mathcal{M}_{b}$, where $K_{n}$ and $K_{n}^{\text {loc }}$ are respectively the Kato and the local Kato classes introduced by Aizenman and Simon [1].

The main results about the problem $\left(\mathcal{P}_{V}\right)$ are the following.

Theorem I. Let $V \in P_{\mathrm{loc}}^{\infty}$. For any $M>1$, there is a constant $b_{0}>0$ such that for each nonnegative $u_{0} \in C^{2}\left(\mathbb{R}^{n}\right)$ satisfying $\left\|u_{0}\right\|_{\infty} \leq b_{0}$, there exists a positive continuous solution $u$ of $\left(\mathcal{P}_{V}\right)$ such that

$$
M^{-1} \int_{\mathbb{R}^{n}} G_{a}(x, t ; y, 0) u_{0}(y) d y \leq u(x, t) \leq M \int_{\mathbb{R}^{n}} G_{a}(x, t ; y, 0) u_{0}(y) d y,
$$

for all $\left.(x, t) \in \mathbb{R}^{n} \times\right] 0, \infty\left[\right.$. Here $G_{a}$ is the fundamental solution of $a \Delta_{x}-\partial / \partial t$ on $\mathbb{R}^{n} \times \mathbb{R}$.

Theorem II. Assume that $V$ is defined on $\left.\mathbb{R}^{n} \times\right] 0, \infty[, V \geq 0$, and the result of Theorem I holds for all $a>0$. Then, we have for all $c>0$,

$$
\sup _{(x, t) \in \mathbb{R}^{n} \times \mathbb{R}} \int_{-\infty}^{t} \int_{\mathbb{R}^{n}} \Gamma_{c}(x, t ; y, s)|V(y, s)| d y d s<\infty
$$

and

$$
\lim _{r \rightarrow 0} \sup _{(x, t) \in A} \int_{t-r}^{t} \int_{|x-y|<\sqrt{r}} \Gamma_{c}(x, t ; y, s)|V(y, s)| d y d s=0,
$$

for any compact subset $A \subset \mathbb{R}^{n} \times \mathbb{R}$. 
By using the same arguments as in [6], we also have the following result about the large-time behavior.

Theorem III. Let $V=V(x) \in P_{\mathrm{loc}}^{\infty}, u_{0} \in C^{2}\left(\mathbb{R}^{n}\right)$ with $0<\beta \leq u_{0} \leq \alpha$ and $\Delta u_{0} \in P_{\mathrm{loc}}^{\infty}$. Then the problem $\left(\mathcal{P}_{V}\right)$ has a global positive solution which converges pointwise to a positive solution of the elliptic problem $a \Delta u+V(x) u^{p}=0$.

In section 2, we prove the continuity of the parabolic potentials $\iint G_{a}|V| d y d s$ when $V \in P_{\text {loc }}^{\infty}$. In section 3, we present an alternative and shorter proof of Theorem I based on a fixed point argument more simple than the Schauder theorem used by Zhang in [5]. In section 4 , we prove Theorem II. In section 5, we characterize the classes $P^{\infty}$ and $P_{\text {loc }}^{\infty}$ in special cases which allow us to give interesting examples of potentials in the class $P_{\mathrm{loc}}^{\infty}$ which do not belong to the class $P^{\infty}$.

Throughout the paper the letter $C$ denotes a positive constant which may vary in value from line to line but not on dependence.

\section{Continuity of parabolic potentials}

In this section we prove the continuity of the potentials $\iint G_{a}|V| d y d s$ when $V \in P_{\text {loc }}^{\infty}$. The continuity is required to establish the global existence result of Theorem I.

For $a>0, x, y \in \mathbb{R}^{n}$ and $t>s$, let

$$
\Gamma_{a}(x, t ; y, s)=\frac{1}{(t-s)^{n / 2}} \exp \left(-a \frac{|x-y|^{2}}{t-s}\right) .
$$

The fundamental solution of $a \Delta_{x}-\partial / \partial t$ on $\mathbb{R}^{n} \times \mathbb{R}$ is given by

$$
G_{a}(x, t ; y, s)= \begin{cases}(4 a \pi)^{-n / 2} \Gamma_{\frac{1}{4 a}}(x, t ; y, s) & \text { if } t>s, \\ 0 & \text { if } t<s .\end{cases}
$$

For $\alpha, \beta \geq 0$ we use the notation $\alpha \vee \beta$ and $\alpha \wedge \beta$ to mean $\max (\alpha, \beta)$ and $\min (\alpha, \beta)$, respectively. We also need to use the inequality

$$
e^{-\theta} \leq \frac{1 \vee(m / e)^{m}}{(1 \vee \theta)^{m}}, \quad \text { for all } \theta \geq 0 \text { and } m>0 .
$$

For $(x, t),(y, s) \in \mathbb{R}^{n} \times \mathbb{R}$, let $d((x, t),(y, s)) \equiv|x-y| \vee|t-s|^{1 / 2}$ be the parabolic distance on $\mathbb{R}^{n} \times \mathbb{R}$. In particular it satisfies the triangle inequality. For $r>0$, let $B((x, t), r)$ be the ball with center $(x, t)$ and radius $r$ with respect to the parabolic distance and $B^{c}((x, t), r)$ its complement. We first prove the following.

Lemma 2.1. Let $a>0$. Then there exist constants $C=C(a)>0$ and $c=c(a)>0$ such that for all $r \in] 0,1\left[,\left(x_{0}, t_{0}\right) \in \mathbb{R}^{n} \times \mathbb{R},(x, t) \in B\left(\left(x_{0}, t_{0}\right), \sqrt{r} / 8\right), t \geq t_{0}\right.$ and $(y, s) \in B^{c}\left(\left(x_{0}, t_{0}\right), \sqrt{r} / 2\right)$, we have

$$
\left|G_{a}(x, t ; y, s)-G_{a}\left(x_{0}, t_{0} ; y, s\right)\right| \leq C\left(\frac{\left|x-x_{0}\right| \vee\left|t-t_{0}\right|^{1 / 2}}{r^{1 / 2}}\right) G_{c}(x, t ; y, s) .
$$

Proof. Case 1. $s \geq t$. The left-hand side term is equal to zero and so the inequality is trivial.

Case 2. $t_{0} \leq s<t$. From the assumptions we have $0<t-s \leq t-t_{0}$ and $|x-y| \vee|t-s|^{1 / 2} \geq 3 \sqrt{r} / 8$. By noting that $G_{a}\left(x_{0}, t_{0} ; y, s\right)=0$ and applying (2.1) 
with $m=1 / 2$, we have

$$
\begin{gathered}
\left|G_{a}(x, t ; y, s)-G_{a}\left(x_{0}, t_{0} ; y, s\right)\right|=\frac{(4 a \pi)^{-n / 2}}{(t-s)^{n / 2}} \exp \left(-\frac{|x-y|^{2}}{4 a(t-s)}\right) \\
\leq C \frac{(t-s)^{1 / 2}}{|x-y| \vee(t-s)^{1 / 2}} \frac{\exp \left(-\frac{|x-y|^{2}}{8 a(t-s)}\right)}{(t-s)^{n / 2}} \leq C \frac{\left(t-t_{0}\right)^{1 / 2}}{r^{1 / 2}} G_{2 a}(x, t ; y, s) .
\end{gathered}
$$

Case 3. $s<t_{0}$. We have

$$
\begin{aligned}
\left|G_{a}(x, t ; y, s)-G_{a}\left(x_{0}, t_{0} ; y, s\right)\right| & \leq\left|G_{a}(x, t ; y, s)-G_{a}\left(x_{0}, t ; y, s\right)\right| \\
+ & \left|G_{a}\left(x_{0}, t ; y, s\right)-G_{a}\left(x_{0}, t_{0} ; y, s\right)\right| .
\end{aligned}
$$

Moreover, we have

$$
\begin{array}{r}
\left|G_{a}(x, t ; y, s)-G_{a}\left(x_{0}, t ; y, s\right)\right| \leq\left|x-x_{0}\right| \sup _{z \in\left[x, x_{0}\right]}\left|\nabla_{z} G_{a}(z, t ; y, s)\right| \\
\leq C\left|x-x_{0}\right| \sup _{z \in\left[x, x_{0}\right]}|z-y| \frac{\exp \left(-\frac{|z-y|^{2}}{4 a(t-s)}\right)}{(t-s)^{n / 2+1}} .
\end{array}
$$

On the other hand, by applying (2.1) with $m=2$, we obtain

$$
|z-y| \frac{\exp \left(-\frac{|z-y|^{2}}{4 a(t-s)}\right)}{(t-s)^{n / 2+1}} \leq \frac{C}{|z-y| \vee(t-s)^{1 / 2}} \frac{\exp \left(-\frac{|z-y|^{2}}{8 a(t-s)}\right)}{(t-s)^{n / 2}}
$$

Moreover, by the triangle inequality of $d$, for $z \in\left[x, x_{0}\right]$, we have

$$
\begin{array}{r}
|z-y| \vee(t-s)^{1 / 2} \geq|x-y| \vee(t-s)^{1 / 2}-|x-z| \\
\geq|x-y| \vee(t-s)^{1 / 2}-\sqrt{r} / 8 \geq \frac{2}{3}|x-y| \vee(t-s)^{1 / 2} \geq \sqrt{r} / 4,
\end{array}
$$

which yields $|z-y|^{2}+t-s \geq \frac{2}{9}\left(|x-y|^{2}+t-s\right)$ and so

$$
\frac{|z-y|^{2}}{t-s} \geq \frac{2}{9} \frac{|x-y|^{2}}{t-s}-\frac{7}{9}
$$

Combining (2.3), (2.4) and (2.5), we obtain

$$
\left|G_{a}(x, t ; y, s)-G_{a}\left(x_{0}, t ; y, s\right)\right| \leq C \frac{\left|x-x_{0}\right|}{r^{1 / 2}} G_{9 a}(x, t ; y, s) .
$$

In the same way, there is a $\theta \in] t_{0}, t[$ such that

$$
\left|G_{a}\left(x_{0}, t ; y, s\right)-G_{a}\left(x_{0}, t_{0} ; y, s\right)\right|=\left|t-t_{0}\right|\left|\frac{\partial}{\partial \theta} G_{a}\left(x_{0}, \theta ; y, s\right)\right| .
$$

Moreover by applying (2.1) with $m=n / 2+2$ and using the inequality $0<\theta-s \leq$ $t-s$, we have

$$
\begin{aligned}
\left|\frac{\partial}{\partial \theta} G_{a}\left(x_{0}, \theta ; y, s\right)\right| & \leq\left(\frac{n}{2}+\frac{\left|x_{0}-y\right|^{2}}{4 a(\theta-s)}\right) \frac{\exp \left(-\frac{\left|x_{0}-y\right|^{2}}{4 a(\theta-s)}\right)}{(\theta-s)^{n / 2+1}} \\
& \leq \frac{C}{\left(\left|x_{0}-y\right| \vee(\theta-s)^{1 / 2}\right)^{n+2}} \exp \left(-\frac{\left|x_{0}-y\right|^{2}}{8 a(t-s)}\right)
\end{aligned}
$$

By noting that $\left|x_{0}-y\right| \vee(\theta-s)^{1 / 2} \geq \frac{2}{3}|x-y| \vee(t-s)^{1 / 2} \geq \sqrt{r} / 4$ and $\frac{\left|x_{0}-y\right|^{2}}{t-s} \geq$ $\frac{2}{9} \frac{|x-y|^{2}}{t-s}-\frac{7}{9}$, it follows that

$$
\left|\frac{\partial}{\partial \theta} G_{a}\left(x_{0}, \theta ; y, s\right)\right| \leq \frac{C}{r} G_{9 a}(x, t ; y, s) .
$$


Combining (2.7) and (2.8), we obtain

$$
\left|G_{a}\left(x_{0}, t ; y, s\right)-G_{a}\left(x_{0}, t_{0} ; y, s\right)\right| \leq C\left(\frac{\left|t-t_{0}\right|}{r}\right)^{1 / 2} G_{9 a}(x, t ; y, s) .
$$

Combining (2.2), (2.6) and (2.9), we obtain the inequality stated in the lemma.

Remark 2.2. If we replace $t \geq t_{0}$ by $t \leq t_{0}$, we obtain the same inequality provided that $G_{c}(x, t ; y, s)$ is replaced by $G_{c}\left(x_{0}, t_{0} ; y, s\right)$.

For $(x, t),\left(x_{0}, t_{0}\right) \in \mathbb{R}^{n} \times \mathbb{R}$, we put

$$
K_{a}\left(x, t ; x_{0}, t_{0}\right)=\int_{-\infty}^{t \vee t_{0}} \int_{\mathbb{R}^{n}}\left|G_{a}(x, t ; y, s)-G_{a}\left(x_{0}, t_{0} ; y, s\right)\right||V(y, s)| d y d s .
$$

We have the following.

Proposition 2.3. Let $V \in P_{\mathrm{loc}}^{\infty}$. Then for all $a>0$,

$$
\lim _{\left|x-x_{0}\right| \vee\left|t-t_{0}\right|^{\frac{1}{2}} \rightarrow 0} K_{a}\left(x, t ; x_{0}, t_{0}\right)=0 .
$$

Proof. Let $\left(x_{0}, t_{0}\right) \in \mathbb{R}^{n} \times \mathbb{R}$ be fixed. Put $A=\bar{B}\left(\left(x_{0}, t_{0}\right), 1\right)$. Since $V \in P_{\mathrm{loc}}^{\infty}$, for $\varepsilon>0$, there exists $r>0$ sufficiently small such that

$$
\sup _{(x, t) \in A} \int_{t-r}^{t} \int_{|x-y|<\sqrt{r}} G_{a}(x, t ; y, s)|V(y, s)| d y d s<\varepsilon .
$$

For $\left|x-x_{0}\right| \vee\left|t-t_{0}\right|^{1 / 2} \leq \sqrt{r} / 8$, we have

$$
\begin{aligned}
K_{a}\left(x, t ; x_{0}, t_{0}\right) & =\int_{-\infty}^{t \vee t_{0}} \int_{\mathbb{R}^{n}}\left|G_{a}(x, t ; y, s)-G_{a}\left(x_{0}, t_{0} ; y, s\right)\right||V(y, s)| d y d s \\
& =\iint_{B\left(\left(x_{0}, t_{0}\right), \sqrt{r} / 2\right)} \ldots d y d s+\iint_{B^{c}\left(\left(x_{0}, t_{0}\right), \sqrt{r} / 2\right)} \ldots d y d s \\
& \equiv I_{1}\left(x, t ; x_{0}, t_{0}\right)+I_{2}\left(x, t ; x_{0}, t_{0}\right) .
\end{aligned}
$$

By (2.10), for $\left|x-x_{0}\right| \vee\left|t-t_{0}\right|^{1 / 2} \leq \sqrt{r} / 8$, we have

$$
\begin{aligned}
I_{1}\left(x, t ; x_{0}, t_{0}\right) \leq & \iint_{B((x, t), \sqrt{r})} G_{a}(x, t ; y, s)|V(y, s)| d y d s \\
& +\iint_{B\left(\left(x_{0}, t_{0}\right), \sqrt{r}\right)} G_{a}\left(x_{0}, t_{0} ; y, s\right)|V(y, s)| d y d s \\
\leq & \varepsilon+\varepsilon=2 \varepsilon .
\end{aligned}
$$

By Lemma 2.1, for $\left|x-x_{0}\right| \vee\left|t-t_{0}\right|^{1 / 2} \leq \sqrt{r} / 8$, we have

$$
I_{2}\left(x, t ; x_{0}, t_{0}\right) \leq C\left(\frac{\left|x-x_{0}\right| \vee\left|t-t_{0}\right|^{1 / 2}}{r^{1 / 2}}\right) N_{c}(V) .
$$

This ends the proof.

Remark 2.4. Similarly, for $(y, s),\left(y_{0}, s_{0}\right) \in \mathbb{R}^{n} \times \mathbb{R}$ and $a>0$, let

$$
K_{a}^{*}\left(y, s ; y_{0}, s_{0}\right)=\int_{s \wedge s_{0}}^{+\infty} \int_{\mathbb{R}^{n}}\left|G_{a}^{*}(y, s ; x, t)-G_{a}^{*}\left(y_{0}, s_{0} ; x, t\right)\right||V(x, t)| d x d t .
$$

If $V \in P_{\mathrm{loc}}^{\infty}$, then $\lim _{\left|y-y_{0}\right| V\left|s-s_{0}\right|^{\frac{1}{2}} \rightarrow 0} K_{a}^{*}\left(y, s ; y_{0}, s_{0}\right)=0$. 


\section{Proof of Theorem I}

For simplicity, we put

$$
h(x, t)=\int_{\mathbb{R}^{n}} G_{a}(x, t ; y, 0) u_{0}(y) d y .
$$

The function $h$ is continuous on $\mathbb{R}^{n} \times\left[0, \infty\left[\right.\right.$ and $0 \leq h \leq\left\|u_{0}\right\|_{\infty}$. Let $C_{b}\left(\mathbb{R}^{n} \times[0, \infty[)\right.$ be the set of all bounded continuous solutions on $\mathbb{R}^{n} \times[0, \infty[$. It is well known that $\left(C_{b}\left(\mathbb{R}^{n} \times\left[0, \infty[),\|\cdot\|_{\infty}\right)\right.\right.$ is a Banach space. For $M>1$, let us define the set

$$
S=\left\{u \in C _ { b } \left(\mathbb{R}^{n} \times\left[0, \infty[): M^{-1} h \leq u \leq M h\right\} .\right.\right.
$$

Clearly $S$ is a nonempty closed subset of $C_{b}\left(\mathbb{R}^{n} \times[0, \infty[)\right.$. We define the integral operator $T$ on $C_{b}\left(\mathbb{R}^{n} \times[0, \infty[)\right.$ by

$$
T u(x, t)=h(x, t)+\int_{0}^{t} \int_{\mathbb{R}^{n}} G_{a}(x, t ; y, s) V(y, s) u^{p}(y, s) d y d s .
$$

Since $u$ is bounded, by Proposition 2.3, $T u \in C_{b}\left(\mathbb{R}^{n} \times[0, \infty[)\right.$. Moreover, as in [5] (see p. 1550), there are constants $C=C(a, p)>0$ and $c=c(a, p)>0$ such that for all $u \in S,|T u-h| \leq M\left(M b_{0}\right)^{p-1} C N_{c}(V) h$. It follows that for $b_{0}$ sufficiently small, $M^{-1} h \leq T u \leq M h$, which means that $T u \in S$ and so $T$ maps $S$ into itself. Moreover for all $u, v \in S$, we clearly have $\|T u-T v\|_{\infty} \leq p\left(M b_{0}\right)^{p-1} C N_{c}(V) \| u-$ $v \|_{\infty}$. In particular for $b_{0}$ small enough, we obtain $\|T u-T v\|_{\infty} \leq \frac{1}{2}\|u-v\|_{\infty}$. Thus $T$ is a $\frac{1}{2}$-Lipschitz mapping from $S$ into itself, and so by the well-known fixed point theorem there exists $u \in S$ such that $T u=u$.

\section{Proof of Theorem II}

Assume that $V$ is defined on $\left.\mathbb{R}^{n} \times\right] 0, \infty[, V \geq 0$ and for all $a>0$ the result of Theorem I holds. By the assumptions, for all $M>0$ there is a constant $b_{0}$ such that for each nonnegative $u_{0} \in C^{2}\left(\mathbb{R}^{n}\right)$ with $\left\|u_{0}\right\|_{\infty} \leq b_{0}$, there exists a solution $u$ of the integral equation

$$
u(x, t)=\int_{\mathbb{R}^{n}} G_{a}(x, t ; y, 0) u_{0}(y) d y+\int_{0}^{t} \int_{\mathbb{R}^{n}} G_{a}(x, t ; y, s) V(y, s) u^{p}(y, s) d y d s
$$

satisfying

$$
M^{-1} \int_{\mathbb{R}^{n}} G_{a}(x, t ; y, 0) u_{0}(y) d y \leq u(x, t) \leq M \int_{\mathbb{R}^{n}} G_{a}(x, t ; y, 0) u_{0}(y) d y .
$$

For $u_{0} \equiv b_{0}$, we have $u(x, t)=b_{0}+\int_{0}^{t} \int_{\mathbb{R}^{n}} G_{a}(x, t ; y, s) V(y, s) u^{p}(y, s) d y d s$ and $M^{-1} b_{0} \leq u(x, t) \leq M b_{0}$, which implies

$$
\sup _{(x, t) \in \mathbb{R}^{n} \times \mathbb{R}} \int_{-\infty}^{t} \int_{\mathbb{R}^{n}} G_{a}(x, t ; y, s)|V(y, s)| d y d s \leq(M-1) M^{p} b_{0}^{1-p}<\infty .
$$

Moreover,

$$
p(x, t) \equiv \int_{-\infty}^{t} \int_{\mathbb{R}^{n}} G_{a}(x, t ; y, s) V(y, s) u^{p}(y, s) d y d s=u(x, t)-b_{0}
$$

is a continuous potential. For $r \in] 0,1[$, let

$$
p_{r}(x, t)=\iint_{B((x, t), \sqrt{r})} G_{a}(x, t ; y, s) V(y, s) u^{p}(y, s) d y d s
$$


and

$$
q_{r}(x, t)=\iint_{B^{c}((x, t), \sqrt{r})} G_{a}(x, t ; y, s) V(y, s) u^{p}(y, s) d y d s .
$$

We will prove that $q_{r}$ is continuous. Let $\left(x_{0}, t_{0}\right) \in \mathbb{R}^{n} \times \mathbb{R}$. For $\left|x-x_{0}\right| \vee\left|t-t_{0}\right|^{1 / 2} \leq$ $\sqrt{r} / 8$, we have

$$
\left|q_{r}(x, t)-q_{r}\left(x_{0}, t_{0}\right)\right| \leq\left(M b_{0}\right)^{p}\left(J_{1}\left(x, t ; x_{0}, t_{0}\right)+J_{2}\left(x, t ; x_{0}, t_{0}\right)\right),
$$

where

$$
J_{1}=\iint_{B^{c}((x, t), \sqrt{r})}\left|G_{a}(x, t ; y, s)-G_{a}\left(x_{0}, t_{0} ; y, s\right)\right| V(y, s) d y d s
$$

and

$$
J_{2}=\iint\left|\mathbf{1}_{B^{c}((x, t), \sqrt{r})}-\mathbf{1}_{B^{c}\left(\left(x_{0}, t_{0}\right), \sqrt{r}\right)}\right| G_{a}\left(x_{0}, t_{0} ; y, s\right) V(y, s) d y d s .
$$

If $|x-y| \vee|t-s|^{1 / 2} \geq \sqrt{r}$, then $\left|x_{0}-y\right| \vee\left|t_{0}-s\right|^{1 / 2} \geq \sqrt{r} / 2$ and so by Lemma 2.1 and (4.1), we obtain

$$
J_{1}\left(x, t ; x_{0}, t_{0}\right) \leq C \frac{\left|x-x_{0}\right| \vee\left|t-t_{0}\right|^{1 / 2}}{r^{1 / 2}} .
$$

On the other hand, by the dominated convergence theorem, we also have

$$
J_{2}\left(x, t ; x_{0}, t_{0}\right) \rightarrow 0 \quad \text { as } \quad\left|x-x_{0}\right| \vee\left|t-t_{0}\right|^{1 / 2} \rightarrow 0 .
$$

Hence $q_{r}$ is continuous. We then have $p_{r}=p-q_{r}$ is continuous and $\lim _{r \rightarrow 0} p_{r}(x, t)=0$. So, by the Dini theorem, $\lim _{r \rightarrow 0} \sup _{(x, t) \in A} p_{r}(x, t)=0$, for any compact subset $A \subset \mathbb{R}^{n} \times \mathbb{R}$. Now by recalling that $u^{p} \geq\left(M b_{0}^{-1}\right)^{p}$, we obtain

$$
\lim _{r \rightarrow 0} \sup _{(x, t) \in A} \iint_{B((x, t), \sqrt{r})} \Gamma_{a}(x, t ; y, s)|V(y, s)| d y d s=0,
$$

for any compact subset $A \subset \mathbb{R}^{n} \times \mathbb{R}$, which completes the proof.

\section{The Classes $P^{\infty}$ and $P_{\text {loc }}^{\infty}$}

In this section we characterize the classes $P^{\infty}$ and $P_{\text {loc }}^{\infty}$ in interesting special cases so that the reader may see the kind of potentials admitted for the class $P_{\mathrm{loc}}^{\infty}$ and not for the class $P^{\infty}$. Let $C_{b}\left(\mathbb{R}^{n} \times \mathbb{R}\right)$ be the set of all bounded continuous functions on $\mathbb{R}^{n} \times \mathbb{R}$ and let $C_{0}\left(\mathbb{R}^{n} \times \mathbb{R}\right)$ be the subset of functions in $C_{b}\left(\mathbb{R}^{n} \times \mathbb{R}\right)$ vanishing at infinity. For a Borel measurable function $V$ on $\mathbb{R}^{n} \times \mathbb{R}$, we put

$$
p_{a}^{V}(x, t)=\int_{-\infty}^{t} \int_{\mathbb{R}^{n}} G_{a}(x, t ; y, s)|V(y, s)| d y d s
$$

and

$$
p_{a}^{* V}(y, s)=\int_{s}^{\infty} \int_{\mathbb{R}^{n}} G_{a}(x, t ; y, s)|V(x, t)| d x d t .
$$

We have the following result.

Proposition 5.1. Let $V \in L^{1}\left(\mathbb{R}^{n} \times \mathbb{R}\right)$. Then

(i) $V \in P_{\mathrm{loc}}^{\infty}$ if and only if for all $a>0$, the potentials $p_{a}^{V}$ and $p_{a}^{* V} \in C_{b}\left(\mathbb{R}^{n} \times \mathbb{R}\right)$.

(ii) $V \in P^{\infty}$ if and only if for all $a>0$, the potentials $p_{a}^{V}$ and $p_{a}^{* V} \in C_{0}\left(\mathbb{R}^{n} \times \mathbb{R}\right)$. 
Proof. For simplicity we write, for $r>0$,

$$
p_{a, r}(x, t)=\iint_{B((x, t), \sqrt{r})} G_{a}(x, t ; y, s)|V(y, s)| d y d s
$$

and

$$
q_{a, r}(x, t)=\iint_{B^{c}((x, t), \sqrt{r})} G_{a}(x, t ; y, s)|V(y, s)| d y d s .
$$

The analogous definitions for the adjoint potentials $p_{a, r}^{*}$ and $q_{a, r}^{*}$ are obvious. We will prove (i). Let $V \in P_{\text {loc }}^{\infty}$. Then $N_{a}(V)<\infty$ for all $a>0$ and by Proposition 2.3 and Remark 2.4, $p_{a}^{V}$ and $p_{a}^{* V}$ are continuous. Hence $p_{a}^{V}$ and $p_{a}^{* V} \in C_{b}\left(\mathbb{R}^{n} \times \mathbb{R}\right)$.

Conversely, assume that for all $a>0, p_{a}^{V}$ and $p_{a}^{* V} \in C_{b}\left(\mathbb{R}^{n} \times \mathbb{R}\right)$. Then $N_{a}(V)<$ $\infty$ for all $a>0$. Moreover, by using the same arguments as in the proof of Theorem II, we have, for all $r>0, q_{a, r}$ is continuous, and so $p_{a, r}=p_{a}^{V}-q_{a, r}$ is continuous. Hence, by the Dini theorem, $\lim _{r \rightarrow 0} \sup _{(x, t) \in A} p_{a, r}(x, t)=0$, for any compact subset $A \subset$ $\mathbb{R}^{n} \times \mathbb{R}$. The same conclusion for $p_{a, r}^{*}$ holds. Thus $V \in P_{\mathrm{loc}}^{\infty}$.

Now we will prove (ii). Assume that $V \in P^{\infty}$. Then by (i), $p_{a}^{V}$ and $p_{a}^{* V} \in$ $C_{b}\left(\mathbb{R}^{n} \times \mathbb{R}\right)$. It remains to prove that $p_{a}^{V}$ and $p_{a}^{* V}$ tend to 0 at infinity. Let $\varepsilon>0$. Then, there exists $r>0$ such that

$$
\sup _{(x, t) \in \mathbb{R}^{n} \times \mathbb{R}} p_{a, r}(x, t)<\varepsilon .
$$

On the other hand, by (2.1), for $|x-y| \vee|t-s|^{1 / 2} \geq \sqrt{r}$, we have

$$
G_{a}(x, t ; y, s)|V(y, s)| \leq C \frac{|V(y, s)|}{\left(|x-y| \vee|t-s|^{1 / 2}\right)^{n}} \leq \frac{C}{r^{n / 2}}|V(y, s)|
$$

with $\iint_{\mathbb{R}^{n} \times \mathbb{R}}|V(y, s)| d y d s<\infty$. Then by the dominated convergence theorem it follows that $\lim _{|(x, t)| \rightarrow \infty} q_{a, r}(x, t)=0$, and so there exists a compact subset $A \subset \mathbb{R}^{n} \times \mathbb{R}$ such that for $(x, t) \in A^{c}$,

$$
q_{a, r}(x, t)<\varepsilon \text {. }
$$

Combining (5.1) and (5.2) it follows that for $(x, t) \in A^{c}, p_{a}^{V}(x, t)<\varepsilon$. Hence $p_{a}^{V} \in C_{0}\left(\mathbb{R}^{n} \times \mathbb{R}\right)$. In the same way we obtain $p_{a}^{* V} \in C_{0}\left(\mathbb{R}^{n} \times \mathbb{R}\right)$.

Conversely assume that for all $a>0, p_{a}^{V}$ and $p_{a}^{* V} \in C_{0}\left(\mathbb{R}^{n} \times \mathbb{R}\right)$. Then, for any $\varepsilon>0$, there exists a compact subset $A \subset \mathbb{R}^{n} \times \mathbb{R}$ such that for $(x, t) \in A^{c}$,

$$
p_{a}^{V}(x, t)<\varepsilon .
$$

Moreover by (i), $V \in P_{\text {loc }}^{\infty}$ and so there is $r>0$ small such that

$$
\sup _{(x, t) \in A} p_{a, r}(x, t)<\varepsilon .
$$

Combining (5.3) and (5.4), we obtain that $\sup _{(x, t) \in \mathbb{R}^{n} \times \mathbb{R}} p_{a, r}(x, t)<2 \varepsilon$. This means that $\lim _{r \rightarrow 0} \sup _{(x, t) \in \mathbb{R}^{n} \times \mathbb{R}} p_{a, r}(x, t)=0$. In the same way, $\lim _{r \rightarrow 0} \sup _{(y, s) \in \mathbb{R}^{n} \times \mathbb{R}} p_{a, r}^{*}(y, s)=0$. Thus $V \in P^{\infty}$.

From Proposition 5.1, we deduce the following characterization of the classes $P_{\mathrm{loc}}^{\infty}$ and $P^{\infty}$ in the time-independent case. 
Corollary 5.2. Let $V=V(x) \in L^{1}\left(\mathbb{R}^{n}\right)$. Then

(i) $V \in P_{\mathrm{loc}}^{\infty}$ if and only if the potential $p^{V}(x) \equiv \int_{\mathbb{R}^{n}} \frac{|V(y)|}{|x-y|^{n-2}} d y \in C_{b}\left(\mathbb{R}^{n}\right)$.

(ii) $V \in P^{\infty}$ if and only if the potential $p^{V}(x) \equiv \int_{\mathbb{R}^{n}} \frac{|V(y)|}{|x-y|^{n-2}} d y \in C_{0}\left(\mathbb{R}^{n}\right)$.

Example 5.3. We will provide examples of functions in $P_{\mathrm{loc}}^{\infty} \backslash P^{\infty}$ in the timeindependent and time-dependent cases.

1) Let $g(x, y)=c_{n}|x-y|^{2-n}$, where $c_{n}=\frac{\Gamma(n / 2-1)}{4 \pi^{n / 2}}$, be the Green function of the Laplacian on $\mathbb{R}^{n}, n \geq 3$. For any integer $m \geq 1$, let $x_{m}=(2 m, 0, \ldots, 0) \in \mathbb{R}^{n}$ and choose $\alpha_{m}>0$ such that $\alpha_{m} g\left(., x_{m}\right)<2^{-m}$ on $B^{c}\left(x_{m}, 1\right)$. Let $q_{m}=1 \wedge$ $\alpha_{m} g\left(., x_{m}\right)$. Clearly $q_{m}$ is a continuous $\Delta$-potential with harmonic support contained in the sphere $S\left(x_{m}, r_{m}\right)$, where $r_{m}$ is the positive real number corresponding to $\alpha_{m} g\left(., x_{m}\right)=1$ on $S\left(x_{m}, r_{m}\right)$ (a simple computation yields $\left.r_{m}=\left(\alpha_{m} c_{n}\right)^{\frac{1}{n-2}}\right)$, and so there is a Borel measure $\sigma_{m}$ on $S\left(x_{m}, r_{m}\right)$ with total mass $\alpha_{m}$ such that $q_{m}(x)=\int_{S\left(x_{m}, r_{m}\right)} g(x, y) \sigma_{m}(d y)$. Let $\varphi_{m} \in C^{\infty}\left(\mathbb{R}^{n}\right), \varphi_{m} \geq 0, \int \varphi_{m}(x) d x=1$ and supp $\varphi_{m} \subset B\left(0, r_{m}\right)$. Consider the convolutions

$$
V_{m}(x)=\sigma_{m} * \varphi_{m}(x) \equiv \int_{S\left(x_{m}, r_{m}\right)} \varphi_{m}(x-y) \sigma_{m}(d y)
$$

and put

$$
p_{m}(x)=\int_{\mathbb{R}^{n}} g(x, y) V_{m}(y) d y=\int_{B\left(0, r_{m}\right)} q_{m}(x-y) \varphi_{m}(y) d y .
$$

Since $q_{m}\left(x_{m}\right)=1, q_{m} \leq 2^{-m}$ on $B^{c}\left(x_{m}, 1\right)$ and $q_{m} \leq 1$ on $B\left(x_{m}, 1\right)$, then $p_{m}$ satisfies the same properties. Moreover $p_{m} \in C^{\infty}\left(\mathbb{R}^{n}\right)$. Now let us take

$$
V(x)=\sum_{m=1}^{\infty} V_{m}(x)
$$

We have $V \in C^{\infty}\left(\mathbb{R}^{n}\right)$ and

$$
\int_{\mathbb{R}^{n}} V(x) d x=\sum_{m=1}^{\infty} \int_{\mathbb{R}^{n}} V_{m}(x) d x=\sum_{m=1}^{\infty} \alpha_{m}<\infty .
$$

Moreover, the potential $p(x)=\int_{\mathbb{R}^{n}} g(x, y) V(y) d y=\sum_{m=1}^{\infty} p_{m}(x) \in C^{\infty}\left(\mathbb{R}^{n}\right), p \leq 2$, and for every integer $m \geq 1, p\left(x_{m}\right) \geq p_{m}\left(x_{m}\right)=1$. This shows that $p \in C_{b}\left(\mathbb{R}^{n}\right) \backslash$ $C_{0}\left(\mathbb{R}^{n}\right)$ and so by Corollary $5.2, V \in P_{\text {loc }}^{\infty} \backslash P^{\infty}$.

2) Let $V=V(x)$ be the function defined in 1). Choose a Borel measurable function $\varphi=\varphi(t)$ on $\mathbb{R}$ with $0<\alpha \leq \varphi \leq \beta$. Here $\alpha$ and $\beta$ are two constants. Clearly $\varphi \in L_{\text {loc }}^{1}(\mathbb{R}) \backslash L^{1}(\mathbb{R})$. Then the function $W(x, t)=V(x) \varphi(t) \in P_{\mathrm{loc}}^{\infty} \backslash P^{\infty}$ with $W$ does not belong to $L^{1}\left(\mathbb{R}^{n} \times \mathbb{R}\right)$.

\section{ACKNOWLEDGMENTS}

I thank Professor W. Hansen for his help in the examples of section 5. 


\section{REFERENCES}

[1] M. Aizenman and B. Simon : Brownian motion and Harnack inequality for Schrödinger operators, Comm. Pure Appl. Math., 37 (1982), 209-273. MR0644024 (84a:35062)

[2] C. Kenig and W.M. Ni : An exterior Dirichlet problem with applications to some nonlinear equations arising in geometry, Amer. J. Math., 106 (1984), 689-702. MR0745147|(85j:35072)

[3] F.H. Lin : On the elliptic equation $D_{i}\left(a_{i j}(x) D_{j} U\right)-k(x) U^{p}=0$, Proc. A.M.S., 95 (1985), 219-266. MR0801327 (86k:35041)

[4] W.M. Ni : On the elliptic equation $\Delta v+K(x) u^{\left(\frac{n+2}{n-2}\right)}=0$, its generalizations and applications in geometry, Indiana Univ. Math. J., 31 (1982), 493-529. MR0662915 (84e:35049)

[5] Q.S. Zhang : Global existence and local continuity of solutions for semilinear parabolic equations, Comm. Part. Diff. Eq., 22 (1997), 1529-1557. MR.1469581 (98f:35075)

[6] Q.S. Zhang and Z. Zhao : Global asymptotic behavior of solutions of a semilinear parabolic equation, Proc. A.M.S., 126, 5 (1998), 1491-1500. MR1458274 (98j:35093)

[7] Z. Zhao: On the existence of positive solutions of nonlinear elliptic equations - A probabilistic potential theory approach, Duke Math. J., 69 (1993), 247-258. MR.1203227 (94c:35090)

Department of Mathematics, Faculty of Sciences of Tunis, Campus Universitaire, 2092 Tunis, Tunisia

E-mail address: Lotfi.Riahi@fst.rnu.tn 